\title{
The pulmonary embolism that wasn't: a case of asymmetric pulmonary arterial flow in Scimitar syndrome
}

\author{
Aaron Ong HJ, ${ }^{1}$ Alwin Tong $\mathrm{YH}^{2}{ }^{2}$ Asmah $\mathrm{O},{ }^{3}$ Norazrulrizal $\mathrm{MN}^{2}$ \\ ${ }^{1}$ Clinical Cardiologist, Department of Cardiology, Penang General Hospital, Ministry of Health, Malaysia \\ ${ }^{2}$ Physician, Department of Medicine, Hospital Tuanku Fauziah, Ministry of Health, Malaysia \\ ${ }^{3}$ Radiologist, Department of Radiology, Hospital Tuanku Fauziah, Ministry of Health, Malaysia
}

\begin{abstract}
A 27-year-old Malaysian woman with prior history of recurrent unexplained episodes of syncope was diagnosed in adulthood with Scimitar syndrome, a rare form of partial anomalous pulmonary venous return (PAPVR). Consequently, she underwent a corrective right pulmonary vein to left atrium baffle surgery. Postoperatively, she was readmitted for pleurisy and haemoptysis, with no evidence of respiratory distress. Subsequent workup with computed tomography of pulmonary angiography (CTPA) revealed the presence of a filling defect in the right main pulmonary artery, mimicking a pulmonary embolism. However, in view of atypical presentation and altered cardiopulmonary flow dynamics, a cardiac magnetic resonance (CMR) angiography imaging was performed demonstrating patent right pulmonary artery on delayed phase acquisition with narrowed surgical baffle. The diagnosis of pulmonary embolism was revised. This case highlights the importance of understanding the pathophysiology and flow mechanics of congenital cardiovascular diseases as well as the diagnostic imaging of choice.
\end{abstract}

\section{KEYWORDS}

Scimitar syndrome, pulmonary embolism, computed tomography pulmonary angiography, cardiac magnetic resonance angiography

\section{BACKGROUND}

Scimitar syndrome is a rare congenital cardiopulmonary anomaly characterised by the presence of partial or complete anomalous right pulmonary venous return into the inferior vena cava. This syndrome is associated with other abnormalities including hypoplastic right lung, anomalous systemic arterial supply to the lung, pulmonary hypertension and atrial septal defect. ${ }^{1}$ The term scimitar refers to the appearance of a curvilinear right pulmonary vein on chest radiographs that resembles a curved Turkish sabre. Three dimensional reconstruction computed tomography of pulmonary angiography (CTPA) or cardiac magnetic resonance (CMR) imaging is the best diagnostic modality, allowing for the delineation of the anomalous drainage as well as associated abnormalities. However, the interpretation of these imaging modalities in the setting of postoperative repair of Scimitar syndrome needs to be carefully examined to avoid potential misdiagnoses. We report one such case of Scimitar syndrome in adulthood who was inadvertently diagnosed with pulmonary embolism post corrective surgery.

\section{CASE REPORT}

A 27-year-old Malaysian female who was premorbidly well had initially presented with multiple episodes of unexplained syncope over many years from 2010 to 2018. Her syncopal episodes were infrequent, occurring primarily during exertion, with no prior premonition. Neurological assessment including magnetic resonance imaging (MRI) of the brain and electroencephalogram (EEG) was unremarkable. Electrocardiogram (ECG) revealed right bundle branch block pattern without other abnormalities. Holter monitoring showed infrequent premature ventricular contraction with no significant burden. However, echocardiography indicated a dilated right atrium and right ventricle. Bubble test performed excluded interatrial shunt. Diagnostic cardiac catheterisation done demonstrated a step up in oxygen saturation from $46.3 \%$ in the superior vena cava (SVC) to $76.2 \%$ in the right atrium and $83.3 \%$ in the main pulmonary artery, indicating the presence of an anomalous pulmonary venous drainage. The mean pulmonary artery pressure was measured at $24 \mathrm{mmHg}$. A computed tomography of pulmonary angiography (CTPA) was arranged to further delineate the pulmonary venous drainage anatomy. This revealed a diagnosis of Scimitar syndrome, a rare form of partial anomalous pulmonary venous return (PAPVR). She had an anomalous right pulmonary vein draining into the infradiaphragmatic inferior vena cava with resulting volume overload leading to dilated right atrium and right ventricle. Three dimensional reconstruction of CTPA showed the anomalous right pulmonary venous drainage in (Fig. 1). In view of her recurrent symptoms and aberrant pulmonary venous anatomy, she was referred to the cardiothoracic team and subsequently underwent corrective right pulmonary vein to left atrium baffle surgery in 2019. Essentially, a baffle was created to direct the flow of the right pulmonary vein from the inferior vena cava to the anatomically correct location at the left atrium. There were no intraoperative complications and postoperative recovery was uneventful.

Within months postoperatively, she represented with pleurisy and an episode of haemoptysis. There were no history of fever, dyspnoea, chronic cough or syncopal episodes. She also did not require supplemental oxygen. A chest x-ray done showed fluffy opacity over the lower zone of the right lung (Fig. 2). Potential differential diagnoses considered were pneumonia, pulmonary haemorrhage and bronchiectasis, with a need to rule out pulmonary embolism. Accordingly, a CTPA done revealed the presence of a filling defect in the right main pulmonary artery, mimicking a large pulmonary embolism (Fig. 3). There were also areas of focal pulmonary haemorrhage over the right lung field. A diagnosis of pulmonary embolism was made and anticoagulation was started. However, in view of her atypical presentation and altered pulmonary circulation dynamics, a discussion with our radiology team on the accuracy of CTPA in this context was made. The CTPA diagnosis of a large pulmonary embolism in an otherwise haemodynamically stable patient with no evidence of respiratory distress seems improbable. Following this, a CMR imaging with delayed phase image acquisition was 
arranged. The CMR demonstrated a patent right pulmonary artery on delayed image acquisition (Fig. 4). However, the surgical baffle was noted to have focal narrowing. The diagnosis of pulmonary embolism was revised. The cause of the pulmonary haemorrhage was attributed to increasing pulmonary venous hypertension due to narrowed surgical baffle. She remains under close follow up with the treating team with the aim for a repeat cardiac catheterisation and stenting of the surgical baffle.

Fig. 1: 3D reconstruction of CTPA (view from posterior) demonstrates the presence of an anomalous right pulmonary vein coursing inferiorly into the infradiaphragmatic inferior vena cava, as opposed to the left atrium.

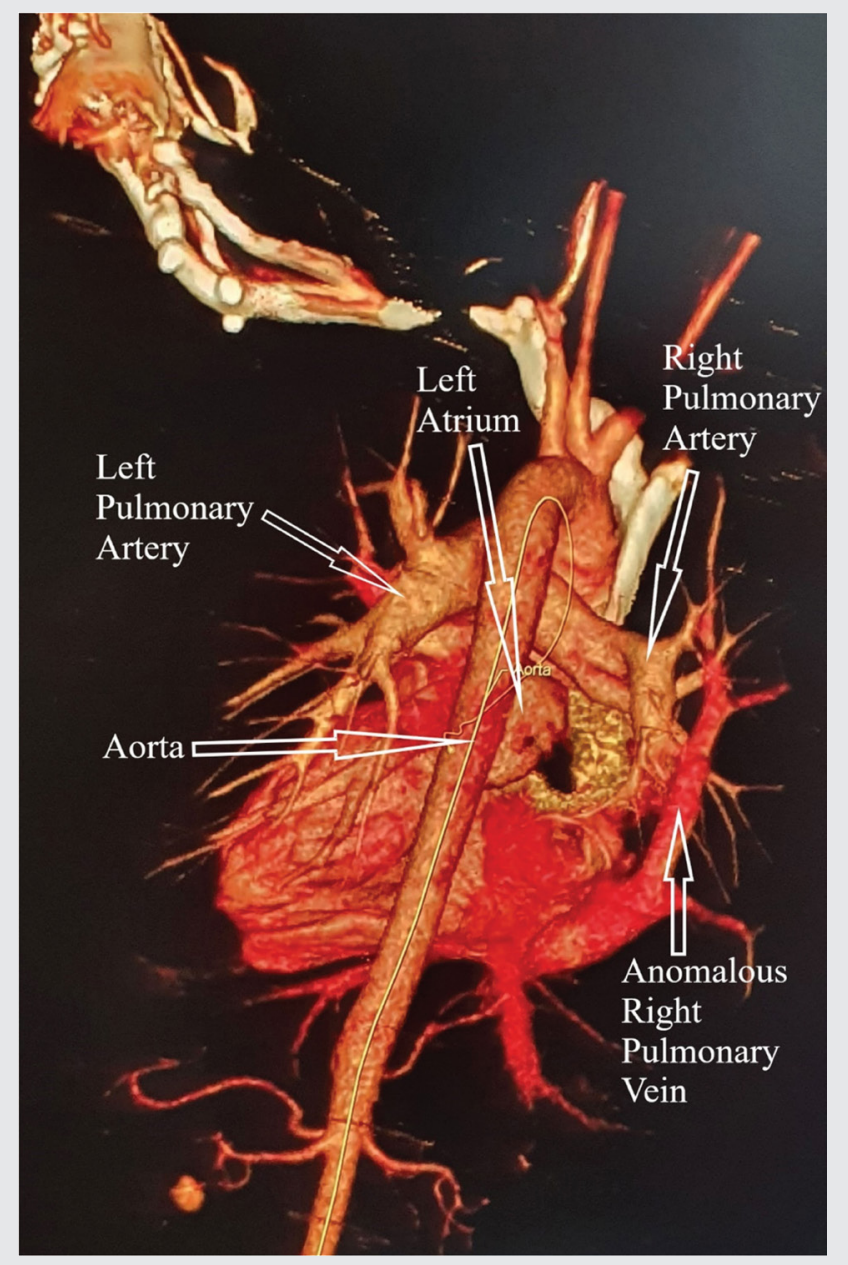

Fig. 2: Erect chest X-ray demonstrates the presence of a curvilinear scimitar along the right heart border. The lower zone of the right lung shows fluffy opacity.

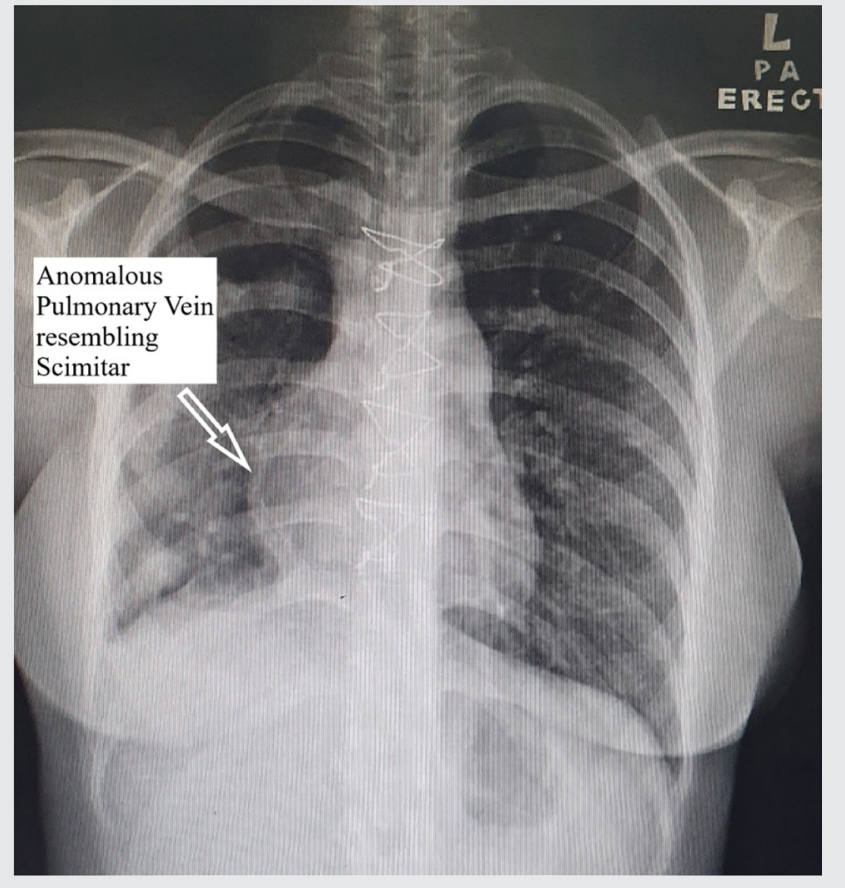

Fig. 3: Computed tomography of pulmonary angiography (CTPA) shows a filling defect in the right main pulmonary artery. The left pulmonary artery is well opacified with contrast.

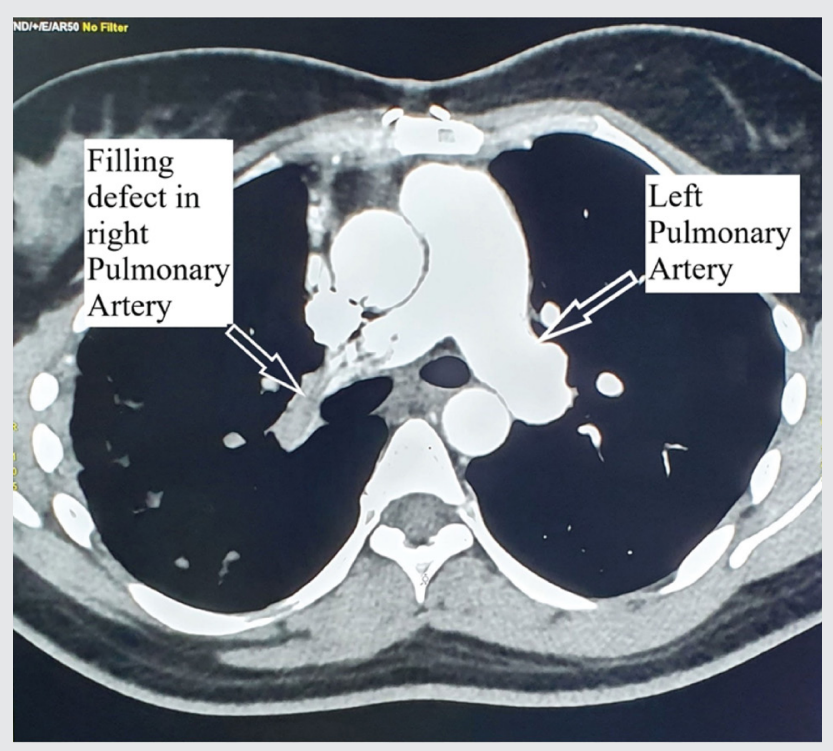




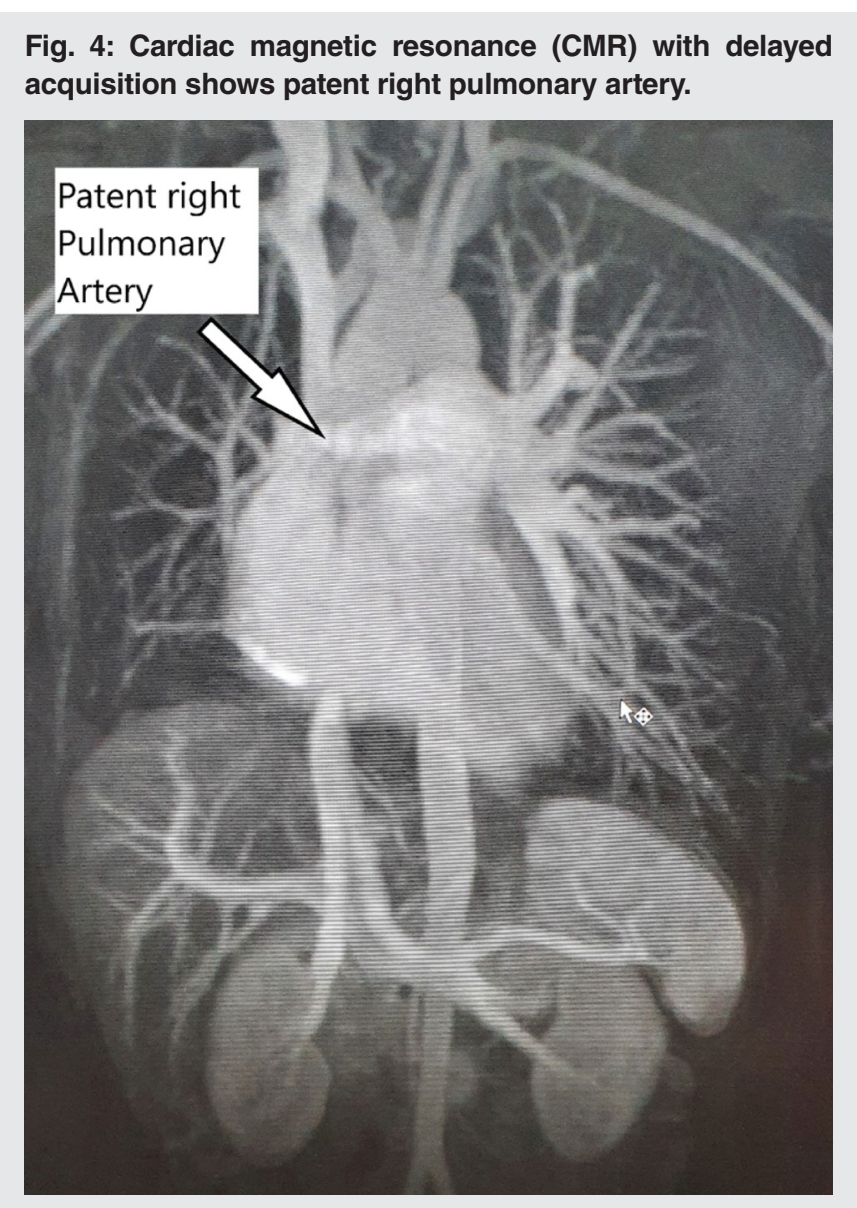

\section{DISCUSSION}

Anomalous pulmonary venous return disorders are congenital defects whereby the right lung drains abnormally via an anomalous vein into the inferior vena cava, right atrium or other systemic veins. When this anomalous vein drains specifically into the infradiaphragmatic inferior vena cava, it is referred to as Scimitar syndrome. ${ }^{2}$ This syndrome is rare, with an incidence of 1-3 per 100,000 live births. ${ }^{3}$ The true incidence may be higher as some patients remain asymptomatic. Patients with this syndrome may be diagnosed early with severe symptoms (infantile form) or late with minimal symptoms or asymptomatic (childhood/adult form). ${ }^{1}$ In infancy, patients may manifest with cyanosis, poor growth, pulmonary hypertension or heart failure. In adulthood, they present with a history of recurrent right lung infection, exertional dyspnoea, signs of pulmonary hypertension or right heart overload. ${ }^{4}$ Syncope as a presenting symptom has been rarely recorded. The diagnosis may be suspected on the presence of a pathognomonic sign on chest X-ray, the Scimitar sign, which is present in $70 \%$ of those with Scimitar syndrome. Computed tomography or CMR can be used to delineate the anomalous drainage.

While majority of asymptomatic patients do not require intervention, indications for surgery in adult patients include recurrent pneumonia, pulmonary hypertension and significant left-to-right shunting. ${ }^{4,5}$ The aim of the surgery is to redirect blood flow from the Scimitar vein to the anatomical left atrium through the creation of a baffle. Specific postoperative complications of Scimitar repair relates primarily to thrombosis, stenosis or occlusion of the surgical baffle. This would result in right sided pulmonary venous hypertension and hence asymmetrically slower right sided pulmonary arterial perfusion relative to the left.

This patient had presented with pleurisy and haemoptysis in the post-operative period, which required the search for an underlying cause. The differential diagnosis considered included pulmonary embolism, pneumonia, bronchiectasis or pulmonary hypertension. CTPA was the gold standard to rule out pulmonary embolism as well as allowing good visualisation of the lung parenchyma. Current CTPA modality allows for a high speed acquisition of images, hence reducing motion and respiration artefacts. However in the postoperative setting of Scimitar syndrome with baffle occlusion, there is asymmetric pulmonary arterial perfusion between the two lungs. This difference in pulmonary arterial flow rate results in a slower opacification of the CTPA contrast to the right pulmonary artery, giving an impression of an apparent filling defect. ${ }^{6}$ This in turn may be misinterpreted as pulmonary embolism. Conversely, CMR imaging allows for dynamic assessment of cardiovascular flow related abnormalities. ${ }^{6}$ While CMR do not have the high spatial resolution of CTPA, it allows for dynamic image acquisition which enables the assessment of perfusion, flow direction and flow rates in the setting of congenital cardiovascular diseases. ${ }^{6}$ With delayed acquisition, the contrast was allowed to opacify the more slowly filling right pulmonary artery, which accounts for the resolution of the apparent filling defect.

\section{CONCLUSIONS}

This case report describes a rare case of an adult patient with Scimitar syndrome who had undergone corrective surgery with subsequent complication of baffle narrowing. The understanding of the flow dynamics and pathophysiology of distinctive congenital cardiovascular diseases are paramount. In turn, this enables the selection of the accurate imaging modality of choice to guide further management.

\section{ACKNOWLEDGEMENT}

We would like to thank the Director General of Health Malaysia for his permission to publish this article.

\section{DECLARATION OF INTERESTS}

The authors have no conflict of interest to declare.

\section{REFERENCES}

1. Ciçek S, Arslan AH, Ugurlucan M, Yildiz Y, Ay S. Scimitar syndrome: the curved Turkish sabre. Semin Thorac Cardiovasc Surg Pediatr Card Surg Annu 2014;17:56-61.

2. Lee M: Isolated and complex scimitar vein anomalies and their differentiation from the meandering right pulmonary vein. Yonsei Med J. 2007, 48:973-980.

3. Dupuis C, Charaf LA, Breviere GM, Abou P, Remy-Jardin M, Helmius G. The "adult" form of the scimitar syndrome. Am J Cardiol. 1992;70:502-07.

4. Gudjonsson U, Brown JW: Scimitar syndrome. Semin Thorac Cardiovasc Surg Pediatr Card Surg Annu. 2006, 9: 56-62.

5. Kamler M, Kerkhoff G, Budde T, Jakob H: Scimitar syndrome in an adult: diagnosis and surgical treatment. Interact Cardiovasc Thorac Surg. 2003, 2: 350-351.

6. Shulman RM, Ayres J, Baffle thrombosis in an adult with remote prior scimitar vein repair mimicking massive pulmonary embolism, Clin Imaging (2014), http://dx.doi.org/10.1016/j.clinimag.2014.03.007 